\title{
Norois
}

Environnement, aménagement, société

Gestion du littoral, risques urbains, ours en Pyrénées

\section{Paskoff (R.), Clus-Auby (C.) - L'érosion des plages : les causes, les remèdes}

Paris/Monaco, Institut Océanographique, coll. « Propos », 2007, 184 p.

\section{Serge Suanez}

\section{(2) OpenEdition}

\section{Journals}

Édition électronique

URL : http://journals.openedition.org/norois/2242

DOI : $10.4000 /$ norois. 2242

ISBN : 978-2-7535-1556-7

ISSN : 1760-8546

Éditeur

Presses universitaires de Rennes

Édition imprimée

Date de publication : 1 novembre 2008

Pagination : 93-94

ISBN : 978-2-7535-0788-3

ISSN : 0029-182X

Référence électronique

Serge Suanez, "Paskoff (R.), Clus-Auby (C.) - L'érosion des plages : les causes, les remèdes », Norois [En ligne], 208 | 2008/3, mis en ligne le 01 novembre 2008, consulté le 23 septembre 2020. URL : http:// journals.openedition.org/norois/2242 ; DOI : https://doi.org/10.4000/norois.2242

(c) Tous droits réservés 
Paskoff (R.), Clus-Auby (C.), 2007. - L'érosion des plages : les causes, les remèdes, Paris/Monaco, Institut Océanographique, coll. "Propos », 184 p.

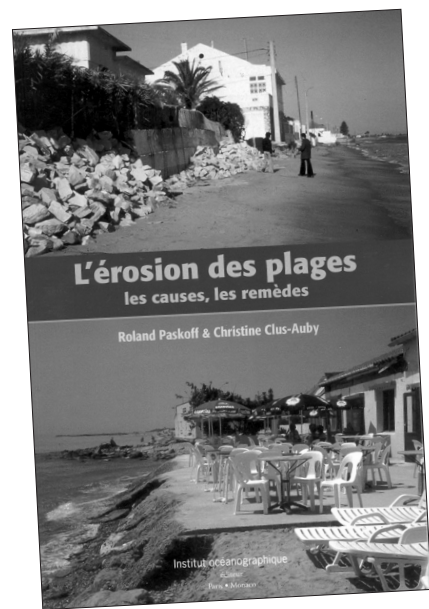

Comme le dit si bien Fernand Verger dans sa préface, l'objectif de l'ouvrage de Roland Paskoff et de Christine Clus-Auby était de réaliser, dans un langage clair et compréhensible par le plus grand nombre, un manuel permettant de comprendre la dynamique morphologique et sédimentaire des plages. Ce livre n'est donc pas un ouvrage scientifique au sens académique du terme, mais bien plus un recueil de connaissances acquises dans le cadre de recherches scientifiques, d'expériences personnelles ou de lectures, le tout permettant d'offrir à l'ensemble des gestionnaires du littoral des éléments de compréhension de l'érosion des plages; à partir de là, on excusera les raccourcis un peu abrupts que l'on trouve dans certaines parties, comme celui visant à caractériser les plages microtidales de plages étroites à pente relativement forte : comment expliquer alors les larges plages très planes de Camargue (?), et vice versa, les plages macrotidales de plages de fond de baie et de golfes: est-ce à dire que toutes les plages de la côte atlantique et de la Manche répondent à cette morphologie (?). De même, à la page 29 la définition qui est donnée de la profondeur de fermeture (closure depth), comme étant égale à la $1 \frac{1}{2}$ longueur d'onde des houles de tempête, manque vraisemblablement de nuance car comment peut-on comprendre deux lignes plus bas qu'elle soit alors comprise entre 8 et $10 \mathrm{~m}$ en Méditerranée et 10 à $20 \mathrm{~m}$ en Atlantique lorsque l'on sait que les périodes maximum de houles de tempête atteignent respectivement 10 et 15 secondes en moyenne.

Pour autant, cet ouvrage présente un réel intérêt. Il permet de renouveler un « guide méthodologique » déjà ancien, qui avait été publié en la matière en 1997 par le ministère de l'Aménagement du Territoire et de l'Environnement (Cataliotti et al., 1997). À ce titre, les nombreux exemples et études de cas qui viennent illustrer le propos apportent de nouveaux éclairages sur les erreurs faites par le passé, et à partir de là, sur les nouvelles orientations prises en matière de lutte contre l'érosion, notamment au niveau des services de l'État.

Les deux premiers chapitres sont respectivement consacrés à la présentation des caractéristiques morphologiques et sédimentaires des plages, et à l'action des agents morphogènes responsables de la mobilité des sédiments. Ainsi, les différentes formes d'accumulation allant des plages sableuses aux cordons de galets sont décrites en fonction de leur morphologie, de leurs caractéristiques sédimentologiques, et des conditions morphodynamiques qui les caractérisent suivant la classification de L. D. Wright et A. D. Short (1984). La dynamique des plages est ensuite abordée en décrivant l'action des forçages naturels que sont les vagues, les courants et le vent. Un rappel est fait sur la notion de bilan sédimentaire des plages en présentant les sources d'apport et de départ des sédiments, à partir desquelles le compartimentage de la côte s'effectue suivant le concept de «cellule hydrosédimentaire».

Le troisième chapitre traite de la thématique centrale de l'ouvrage : l'érosion des plages. À partir de nombreux exemples, les auteurs décrivent les mécanismes à l'origine du recul des côtes d'accumulation. Les causes naturelles telles que la baisse des apports sédimentaires à la côte depuis la fin de la transgression holocène, aggravée par l'élévation actuelle du niveau marin, sont tout d'abord analysées. Une large part est ensuite consacrée à l'action anthropique telle que la construction des barrages le 
long des fleuves, les extractions de sédiments, ou encore la mise en place d'ouvrages littoraux, intervenant dans le dysfonctionnement hydrodynamique et sédimentaire des plages. Les auteurs rappellent que le suivi topo-morphologique visant à suivre et à quantifier le recul des plages reste un préalable essentiel à la gestion de l'érosion, et qu'en la matière, la France reste un des pays européens le plus en retard.

Les trois derniers chapitres traitent des moyens mis en œuvre pour lutter contre l'érosion en insistant respectivement sur les aspects techniques, législatifs et sur la philosophie à adopter pour arriver à une " gestion vertueuse » du littoral. Ainsi, les auteurs analysent de façon méticuleuse les différentes techniques d'ingénierie utilisées pour contenir l'érosion du littoral en insistant bien sur les inconvénients et les avantages de chaque système. Une attention particulière est portée sur les effets négatifs des ouvrages durs en enrochements encore trop largement répandus, en montrant bien que des techniques alternatives telles que les systèmes by-pass, le drainage des plages, ou encore le rechargement des plages, peuvent apparaître plus efficaces et moins dommageables d'un point de vue environnemental. Les auteurs insistent également sur le rôle important des dunes bordières qui restent le premier rempart naturel contre l'assaut des vagues, et qu'à ce titre, une meilleure gestion et préservation de ces milieux permettrait de limiter les processus érosifs. Après avoir souligné l'extraordinaire imbrication des propriétés foncières et des responsabilités réglementaires de la frange littorale, l'ouvrage se termine en exposant les grandes lignes philosophiques d'une gestion que les auteurs qualifient de «vertueuse ». Elle doit avant tout s'appuyer sur un travail d'observation et de mesures visant à quantifier et à comprendre les processus morphodynamiques de manière à orienter du mieux possible les choix d'intervention. Des principes qu'il faudrait suivre mais qui ne le sont pas toujours par les gestionnaires; souhaitons que cet ouvrage puisse les en convaincre, c'est en tout cas ce que fut le vœu de Roland Paskoff et Christine Clus-Auby lorsqu'ils entreprirent la rédaction de ce manuel.

Cataliotti (D.), Michel (P.), Levoy (F.) (dir.), 1997. - La défense des côtes contre l'érosion marine. Pour une approche globale et environnementale, Paris, ministère de l'Aménagement du Territoire et de l'Environnement (Direction de la Nature et des Paysages), 142 p.

Wright (L. D.), ShORT (A. D.), 1984. - "Morphodynamic variability of surf zones and beaches: a synthesis", Marine Geology, Vol. 56, n 1/4, 93-118. 